\title{
Efficacy of alogliptin in preventing non-alcoholic fatty liver disease progression in patients with type 2 diabetes
}

\author{
TSUYOSHI MASHITANI ${ }^{1,2}$, RYUICHI NOGUCHI ${ }^{1}$, YASUSHI OKURA ${ }^{1}$, TADASHI NAMISAKI ${ }^{1}$, AKIRA MITORO $^{1}$, \\ HITOSHI ISHII ${ }^{2}$, TOSHIYA NAKATANI ${ }^{3 *}$, EIRYO KIKUCHI ${ }^{3 *}$, HIROTO MORIYASU $^{4 *}$, MASAMI MATSUMOTO $^{4 *}$, \\ SHINYA SATO $^{5^{*}}$, TATSUICHI AN ${ }^{5^{*}}$, HIROSHI MORITA $^{6^{*}}$, SIGEYUKI AIZAWA $^{7 *}$, YASUNORI TOKUOKA $^{8^{*}}$, \\ MASATOSHI ISHIKAWA ${ }^{9}$, YOSHINOBU MATSUMURA ${ }^{9 *}$, HIROMASA OHIRA $^{10^{*}}$, \\ ATSUKO KOGURE $^{11^{*}}$, KAZUHIRO NOGUCHI ${ }^{1{ }^{*}}$ and HITOSHI YOSHIJI ${ }^{1 *}$
}

\begin{abstract}
${ }^{1}$ Third Department of Internal Medicine, ${ }^{2}$ Department of Diabetology, Nara Medical University, Kashihara, Nara 634-8521;
${ }^{3}$ Department of Gastroenterology, Nara Prefecture General Medical Center, Nara 631-846; ${ }^{4}$ Department of Gastroenterology,

Nara Prefectural Gojo Hospital, Gojo, Nara 637-8511; ${ }^{5}$ Department of Gastroenterology, Belland General Hospital, Sakai, Osaka 599-8247; ${ }^{6}$ Department of Internal Medicine, Takamiya Hospital, Tenri, Nara 632-0052; ${ }^{7}$ Department of Gastroenterology,

Nara Prefecture Western Medical Center, Nara 636-0802; ${ }^{8}$ Department of Internal Medicine, Kokuho Chuo Hospital,

Nara 636-0302; ${ }^{9}$ Department of Gastroenterology, Ishinkai Yao General Hospital, Yao, Osaka 581-0036; ${ }^{10}$ Department

of Gastroenterology and Rheumatology, School of Medicine, Fukushima Medical University, Fukushima 960-1295;

${ }^{11}$ Department of Gastroenterology, Fujita General Hospital, Fukusima 969-1793, Japan
\end{abstract}

Received August 19, 2015; Accepted December 12, 2015

DOI: 10.3892/br.2016.569

\begin{abstract}
Non-alcoholic fatty liver disease (NAFLD) represents one of the most common causes of chronic liver disease worldwide and is characterized by chronic liver inflammation and fibrosis leading to cirrhosis and increased risk of liver cancer in a proportion of patients. Effective antifibrotic agents have yet to be approved for the treatment of NAFLD. The present study aimed to evaluate the efficacy of dipeptidyl peptidase 4 inhibitors (DPP4-I) in the prevention of NAFLD progression in NAFLD patients with type 2 diabetes. The study was a single arm, multi-centre, non-randomised study of NAFLD patients with type 2 diabetes. NAFLD was diagnosed according to ultrasonographic findings. All the patients received $25 \mathrm{mg} /$ day of alogliptin for 12 months. The efficacy of alogliptin in preventing NAFLD progression was assessed using overall NAFIC scores [non-alcoholic steatohepatitis (NASH), ferritin, insulin and type IV collagen 7S] and individual component scores according to baseline haemoglobin A1c (HbA1c) levels. Of the 39 patients enrolled in the study, 16 patients $(40.3 \%$ ) had NAFIC scores $>2$ points, indicating the presence of NASH. NAFIC scores markedly
\end{abstract}

Correspondence to: Dr Tsuyoshi Mashitani, Third Department of Internal Medicine, Nara Medical University, 840 Shijo-cho, Kashihara, Nara 634-8521, Japan

E-mail: mashiyan@gmail.com

*On behalf of the NGF Study Group

Key words: non-alcoholic fatty liver disease, diabetes, dipeptidyl peptidase 4 inhibitor decreased following 12 months of alogliptin administration, but remained $>2$ points in 10 patients, indicating that NASH may have persisted in these patients. The relative risks for persistent NASH were 4.92 (95\% confidence interval, 0.61-40.0) in the highest HbA1c tertile group compared with those in the lowest group. However, no statistically significant linear trend was observed across all HbAlc categories $(\mathrm{P}=0.145)$. DPP4-I may have efficacy against NAFLD progression in patients with type 2 diabetes with relatively lower HbA1c levels. DPP4-I may represent a potential new therapeutic strategy for the prevention of disease progression in NAFLD patients with type 2 diabetes.

\section{Introduction}

Non-alcoholic fatty liver disease (NAFLD) is one of the most common causes of chronic liver disease worldwide (1). NAFLD encompasses a wide spectrum of liver diseases ranging from simple steatosis to non-alcoholic steatohepatitis, which may progress to liver cirrhosis in $\leq 30 \%$ patients, potentially leading to decompensated liver disease (2). As liver fibrosis progresses over a long period of time, therapies should be tolerable and safe over decades, with good targeting to the liver and few adverse effects on other organs.

However, no anti-fibrotic agents have yet been approved for clinical practice (3). Several studies have evaluated the efficacy of dipeptidyl peptidase 4 inhibitor (DPP4-I) administration in the treatment of NAFLD and non-alcoholic steatohepatitis (NASH) patients with type 2 diabetes. In NAFLD patients, DPP4-I administration has been shown to decrease serum alanine aminotransferase (ALT) levels in a positive correlation with haemoglobin Alc (HbA1c) levels (4), and ameliorate liver enzyme abnormalities and hepatocyte 
ballooning in NASH patients (5). Although our previous study reported DPP4-I as a potential new therapeutic agent against liver fibrosis in an experimental model of liver fibrosis via suppression of activated hepatic stellate cell (HSC) proliferation and collagen synthesis (6), the efficacy of DPP4-I for the prevention of NAFLD progression in clinical settings remains to be elucidated.

The aim of the present study was to evaluate the efficacy of DPP4-I in the prevention of NAFLD progression according to decreases in NAFIC scores. We further hypothesize that, in a similar manner to baseline HbA1c levels being a strong predictor of HbAlc change, the efficacy of DPP4-I in preventing NAFLD progression would be more significant in patients with higher HbAlc levels compared to those with lower levels.

\section{Materials and methods}

Study design. The study was a single arm, multi-center, non-randomized study of NAFLD patients with type 2 diabetes that were recruited from 8 centers in Japan between June 2012 and December 2013. All the patients received $25 \mathrm{mg}$ /day of alogliptin (Takeda, Osaka, Japan) for 12 months.

Patients. Patients enrolled were previously diagnosed with type 2 diabetes according to the diagnostic criteria of the Japan Diabetes Society (JDS) with ALT levels $>30$ IU/L, which is the cut-off level used to screen for NAFLD $(7,8)$. Patients with a current daily alcohol intake of $>20 \mathrm{~g}$ or with known liver disease of other aetiology were excluded. Following confirmation of eligibility, receipt of informed consent and screening procedures, eligible patients were administered alogliptin and followed up over a 1-year period. NAFLD was defined according to characteristic ultrasonographic findings, such as increased hepatorenal contrast or enhanced liver brightness (9). All the patients provided written consent to participate in the study. The protocols used were approved by the Ethics Committee of Nara Medical University (Nara, Japan; UMIN000008068) and other facilities.

Data collection. On the date of alogliptin treatment initiation, patients underwent laboratory tests, routine medical history inquiry and physical examinations, including age, gender and body weight and medical history. Laboratory tests at baseline included overnight fasting measurements of aspartate aminotransferase, ALT, $\gamma$-glutamyltranspeptidase, total cholesterol, triglyceride, high-density lipoprotein cholesterol, fasting plasma glucose, HbAlc, immunoreactive insulin (IRI), ferritin, hyaluronic acid, type IV collagen $7 \mathrm{~S}$ and type III procollagen-N-peptide. These parameters were measured by the standard techniques used in clinical chemistry laboratories (SRL, Tokyo, Japan). One year after registration, IRI, ferritin and type IV collagen 7S levels were obtained for the estimation of NAFIC scores. HbA1c levels were expressed in accordance with the National Glycohemoglobin Standardization Program, as recommended by the JDS (10).

Study outcome. One year after registration, the NAFIC (NASH, ferritin, insulin and type IV collagen 7S) score and each individual variable was used to evaluate the efficacy of alogliptin in the prevention of NAFLD progression in all the included patients.

NAFIC score. The original NAFIC score is a simple clinical scoring system allowing the differentiation of NASH from NAFLD using a cut-off score of 2 . The NAFIC score is a weighted sum of three clinical variables: Serum ferritin $\geq 200 \mathrm{ng} / \mathrm{ml}$ (female) or $\geq 300 \mathrm{ng} / \mathrm{ml}$ (male), 1 point; serum fasting insulin $\geq 10 \mathrm{IU} / \mathrm{ml}, 1$ point; and serum type IV collagen $7 \mathrm{~S} \geq 5.0 \mathrm{ng} / \mathrm{ml}, 2$ points (11).

Statistical analyses. All the variables are expressed as medians and inter quartile ranges. Differences between the groups were evaluated using the unpaired Student's t-test for normally distributed variables and the Mann-Whitney U test for variables with skewed distributions. The trend test was used to evaluate differences between category variables. Relative risk regression analyses were performed to estimate relative risks [95\% confidence interval (CI)] of persistence of NAFIC scores of $>2$ points at the end of the study (i.e. possible persistence of $\mathrm{NASH}$ ) in comparison with the lowest tertile of HbA1c levels as a reference group. All the reported P-values were 2-sided and $\mathrm{P}<0.05$ was considered to indicate a statistically significant difference. All the analyses were performed using Stata/MP version 13.0 (Stata Corporation, College Station, TX, USA).

\section{Results}

Patients. A total of 59 patients were enrolled in the study between June 2012 and December 2013. A total of 20 patients were excluded due to drop out prior to completion (5 patients) and absence of blood examination results (15 patients). The remaining 39 patients met the inclusion criteria and were included in the analysis. Table I shows the patient demographics and laboratory data according to the NAFIC scores. The median patient age, HbAlc level and body mass index were 61 years, $6.8 \%(53.0 \mathrm{mmol} / \mathrm{mol})$ and $28.6 \mathrm{~kg} / \mathrm{m}^{2}$, respectively. In 16 patients $(41.0 \%)$, the NAFIC score was $>2$ points, indicating the presence of NASH in $41.0 \%$ of patients with type 2 diabetes with ultrasonographic fatty liver and ALT levels of $>30 \mathrm{U} / 1$.

Changes in NADIC scores. Fig. 1A shows the changes in the NAFIC scores following treatment with alogliptin. NAFIC scores were significantly decreased at 52 weeks after the initiation of DPP4-I therapy. Fig. 1B shows NAFIC score changes according to baseline NAFIC scores. NAFIC scores decreased in 13 patients and remained $>2$ points in 10 patients, indicating NASH had possibly persisted in these patients. NAFIC scores had increased in only 1 patient at the end of the study compared to baseline scores.

Subsequently, the individual components of the NAFIC score at the baseline and the end of the study were evaluated, stratified according to the baseline HbAlc tertile (Table II). No significant changes in fasting IRI or type 4 collagen $7 \mathrm{~S}$ levels were observed; however, ferritin levels were significantly decreased after 52 weeks administration of DPP4-I. Stratified analysis according to the HbAlc tertile demonstrated significant changes in the ferritin levels in the lowest HbA1c category only. 
Table I. Baseline characteristics of the participants stratified by the NAFIC score.

\begin{tabular}{|c|c|c|c|}
\hline Characteristics & Total $(n=39)$ & NAFIC score 0 to $1(n=23)$ & NAFIC score 2 to $4(n=16)$ \\
\hline Age (years) & $61(52-66)$ & $60(52-64)$ & $62(49-66)$ \\
\hline Body mass index, $\mathrm{kg} / \mathrm{m}^{2}$ & $28.6(26.9-31.2)$ & $28.2(21.9-31.2)$ & $30.1(27.7-33.9)$ \\
\hline Female, $\%$ & 51.3 & 52.2 & 50.0 \\
\hline PLT, $10^{4} \mu 1$ & $19.5(17.1-21.8)$ & $19.6(17.4-25.0)$ & $18.7(11.1-21.4)$ \\
\hline AST, IU/1 & $40(29-53)$ & $31(27-46)$ & $46(37-61)$ \\
\hline ALT, IU/1 & $49(38-66)$ & $47(34-64)$ & $55(42-68)$ \\
\hline$\gamma \mathrm{GTP}, \mathrm{IU} / 1$ & $55(38-86)$ & $50(32-77)$ & $72(44-106)$ \\
\hline $\mathrm{HbA} 1 \mathrm{c}, \%$ & $6.8(6.4-7.9)$ & $6.8(6.4-7.5)$ & $7.1(6.3-8.0)$ \\
\hline $\mathrm{FPG}, \mathrm{mg} / \mathrm{dl}$ & $130(115-159)$ & $130(112-154)$ & $138(122-172)$ \\
\hline Fasting IRI, $\mu \mathrm{U} / \mathrm{ml}$ & $12.6(6.5-19.3)$ & $10.4(5.8-16.5)$ & $17.3(12.0-25.9)$ \\
\hline HOMA-IR & $4.4(2.5-7.7)$ & $2.8(1.9-5.2)$ & $7.0(4.2-9.9)$ \\
\hline $\mathrm{TG}, \mathrm{mg} / \mathrm{dl}$ & $176(123-227)$ & $183(125-213)$ & $172(114-256)$ \\
\hline HDL-C, mg/dl & $46(41-53)$ & $45(41-53)$ & $51(42-54)$ \\
\hline $\mathrm{TC}, \mathrm{mg} / \mathrm{dl}$ & $186(168-207)$ & $182(167-202)$ & $193.5(170-211)$ \\
\hline Ferritin, ng/ml & $155.0(47.0-342.0)$ & $99.3(43.3-179.0)$ & $314.0(52.1-380.0)$ \\
\hline Hs-CRP & $1,010(568-1,680)$ & $1,135(471-2,110)$ & $938(680-1,585)$ \\
\hline Hyaluronic acid, ng/ml & $57(24-86)$ & $34(24-81)$ & $75(28-113)$ \\
\hline $\mathrm{P}-3-\mathrm{P}, \mathrm{U} / \mathrm{ml}$ & $0.53(0.49-0.65)$ & $0.50(0.43-0.59)$ & $0.63(0.51-0.72)$ \\
\hline Type 4 collagen $7 \mathrm{~S}, \mathrm{ng} / \mathrm{ml}$ & $3.7(3.1-5.2)$ & $3.3(2.6-3.7)$ & $5.4(4.5-6.7)$ \\
\hline \multicolumn{4}{|l|}{ OADs use, $\%$} \\
\hline Sulfonylurea & 5.1 & 0.0 & 12.5 \\
\hline Thiazolidinedione & 5.1 & 6.3 & 4.4 \\
\hline Metformin & 2.6 & 4.4 & 0.0 \\
\hline$\alpha$-glucosidase inhibitor & 2.6 & 0.0 & 6.3 \\
\hline Hypertension, \% & 18.0 & 21.7 & 12.5 \\
\hline Dyslipidemia, \% & 18.0 & 13.0 & 25.0 \\
\hline Smoker, \% & 15.4 & 13.0 & 18.8 \\
\hline
\end{tabular}

Data are median values (interquartile range) for continuous variables and \% for categorical variables. ALT, alanine aminotransferase; AST, aspartate aminotransferase; FPG, fasting plasma glucose; $\gamma$ PG, $\gamma$-glutamyltranspeptidase; HbA1c, haemoglobin A1c; HDL-C, high-density lipoprotein cholesterol; HOMA-IR, homeostasis model assessment of insulin resistance; Hs-CRP, high-sensitivity C-reactive protein; IRI, immunoreactive insulin; P-3-P, type III procollagen-Npeptide; PLT, platelet count; TC, total cholesterol; TG, triglyceride; OADs, oral antidiabetic agents.

Table II. Changes in fasting IRI, type 4 collagen $7 \mathrm{~S}$ and ferritin stratified according to baseline HbA1c levels.

\begin{tabular}{|c|c|c|c|c|}
\hline \multirow[b]{2}{*}{ Changes } & \multicolumn{4}{|c|}{$\mathrm{HbA} 1 \mathrm{c}$ range, $\%$} \\
\hline & $5.5-6.5(n=14)$ & $6.6-7.2(n=12)$ & 7.3-11.8 $(n=13)$ & Total \\
\hline \multicolumn{5}{|c|}{ Fasting IRI, $\mu \mathrm{U} / \mathrm{ml}$} \\
\hline Month 0 & $12.7(6.1-48.6)$ & $11.5(6.5-17.2)$ & $13.6(11.5-19.3)$ & $12.6(6.5-19.3)$ \\
\hline Month 12 & $9.6(8.0-17.4)$ & $9.3(6.2-23.1)$ & $12.1(9.1-16.0)$ & $10.4(8.0-17.7)$ \\
\hline P-value & 0.6378 & 0.4328 & 0.1330 & 0.5029 \\
\hline \multicolumn{5}{|c|}{ Type 4 collagen $7 \mathrm{~S}, \mathrm{ng} / \mathrm{ml}$} \\
\hline Month 0 & $3.7(2.8-4.7)$ & $3.7(2.6-4.8)$ & $4.8(3.5-6.8)$ & $3.7(3.1-5.2)$ \\
\hline Month 12 & $3.4(3.3-3.7)$ & $3.6(3.1-4.0)$ & $3.7(3.3-4.5)$ & $3.7(3.3-4.5)$ \\
\hline $\mathrm{P}$-value & 0.2547 & 0.5828 & 0.8887 & 0.6249 \\
\hline \multicolumn{5}{|c|}{ Ferritin, ng/ml } \\
\hline Month 0 & $124.7(85.2-205.0)$ & $211.5(53.0-380.0)$ & $155.0(31.5-286.0)$ & $155.0(47.0-342.0)$ \\
\hline Month 12 & $100.7(54.5-137.0)$ & $137.0(40.3-251.5)$ & $102.0(19.0-251.0)$ & $108.0(36.0-191.0)$ \\
\hline P-value & 0.0035 & 0.0844 & 0.0869 & 0.0003 \\
\hline
\end{tabular}

Data are the median values (interquartile range). HbA1c, haemoglobin A1c; IRI, immunoreactive insulin. 
Table III. Association between the possibly persistent NASH at the end of the study and baseline glycemic control.

\begin{tabular}{llll}
\hline & \multicolumn{3}{c}{ HbA1c tertiles } \\
\cline { 2 - 4 } Possibly persistent NASH & $\begin{array}{c}\text { First } \\
(\mathrm{n}=201)\end{array}$ & $\begin{array}{c}\text { Second } \\
(\mathrm{n}=168)\end{array}$ & $\begin{array}{c}\text { Third } \\
(\mathrm{n}=151)\end{array}$ \\
\hline RR for possibly persistent NASH (crude) & Reference & $1.17(0.28-4.83)$ & $\begin{array}{c}\text { P-value } \\
\text { for trend }\end{array}$ \\
RR for possibly persistent NASH (model 1) & Reference & $1.29(0.14-11.62)$ & $4.92(0.52-6.15)$ \\
$0.40 .0)$
\end{tabular}

Model 1 was adjusted for age, gender and body mass index. HbA1c, haemoglobin A1c; NASH, non-alcoholic steatohepatitis; RR, relative risk.

A

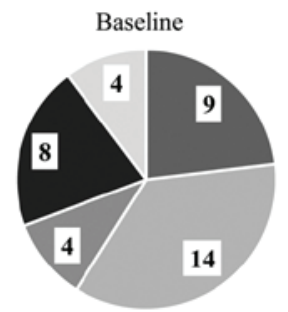

NAFIC score $=0=1=2=3=4$

$\mathbf{B}$

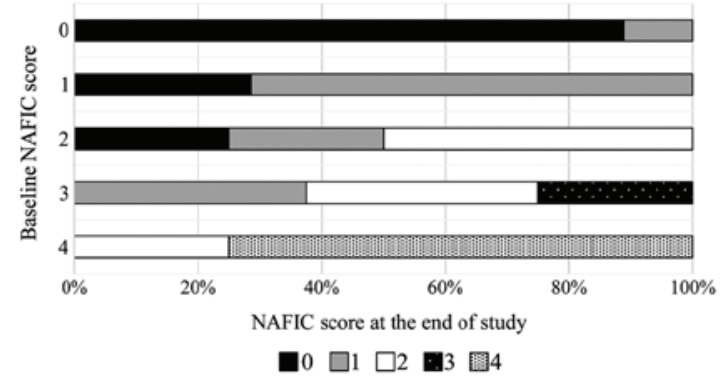

Figure 1. Changes in NAFIC scores. (A) The changes in the NAFIC scores following treatment with alogliptin. NAFIC scores were significantly decreased at 52 weeks after the initiation of dipeptidyl peptidase 4 inhibitors (DPP4-I) therapy. (B) The NAFIC score changes according to baseline NAFIC scores. NAFIC scores decreased in 13 patients and remained $>2$ points in 10 patients.

NASH and HbAlc tertiles. The association between possibly persistent NASH at the end of the study and HbA1c tertiles are shown in Table III. The relative risks for possibly persistent NASH was 4.92 (95\% CI, 0.61-40.0) in the highest HbA1c tertile group compared to the reference category of the lowest HbAlc tertile. However, no statistically significant linear trend was observed across all HbA1c categories $(\mathrm{P}=0.145)$.

\section{Discussion}

To the best of our knowledge, this is the first clinical study to demonstrate the efficacy of administering DPP4-I for 12 months in order to decrease serum ferritin levels. According to the NAFIC scores, this would prevent the progression of NAFLD in patients with type 2 diabetes. The effects were observed only among patients in the lower HbA1c tertile at baseline, which was different from the efficacy in order to use for lowering HbAlc level. Therefore, we speculate that the reduction in oxidative stress, resulting from a decrease in serum ferritin levels, suppressed the extent of liver injury induced by inflammatory cytokines in NAFLD patients with early type 2 diabetes. DPP4-I may therefore be efficient in preventing the progression of NAFLD to NASH in NAFLD patients with early type 2 diabetes.

A number of previous studies have reported the effect of DPP4-I on liver dysfunction in NAFLD patients. Iwasaki et al (12) first reported that 4 months of sitagliptin administration resulted in improved liver enzyme abnormalities in NAFLD patients with type 2 diabetes. Yilmaz et al (13) reported the effect of DPP4-I in patients with biopsy-proven NASH with type 2 diabetes. This study demonstrated that administration of sitagliptin for 12 months ameliorated liver enzyme abnormalities and hepatocyte ballooning in patients whose body weight decreased during the study period. Fukuhara et al (4) reported that administration of sitagliptin for 12 months in patients with biopsy-proven NAFLD with type 2 diabetes improved the liver enzyme abnormalities in parallel with decreases in HbA1c levels. However, the present study did not evaluate the association between changes in body weight, HbA1c levels and NAFIC scores during the study period. This study demonstrated that administration of DPP4-I for 12 months significantly reduced NAFIC scores in NAFLD patients with type 2 diabetes. Furthermore, in patients in the highest HbA1c tertile, NAFIC scores were observed that had remained $>2$ points during the study period, indicating the persistence of NASH. Self-care activity in patients with lower HbA1c levels was known to be higher compared to those with higher HbA1c levels (14); therefore, patients with lower HbAlc levels may have had ideal lifestyles and body weights preventing worsening of HbAlc levels.

In the liver, DPP4 is expressed on the surface of HSCs and may contribute to activated HSC-induced ECM accumulation (15). Kaji et al (6) reported that DPP4-I inhibited liver fibrosis and production of hepatic transforming growth factor- $\beta 1$ (TGF- $\beta 1$ ), along with attenuation of $\alpha$-smooth muscle actin-positive activated HSCs. These results indicate that the suppression of activated HSC function may underlie the anti-fibrotic effect of DPP4-I. As high glucose levels and high insulin levels stimulate the proliferation of activated HSCs in a dose-dependent manner (16), DPP4-I may be more effective against NASH progression in comparatively low glucose conditions. These experimental studies may explain the greater efficacy of DPP4-I in the prevention of NASH progression in patients in the lower HbA1c category in the present study.

Reductions in serum ferritin levels were observed only among NAFIC score components. Experimental models have demonstrated that iron increases hepatocyte apoptosis and contributes to the development of fibrosis directly and indirectly via induction of TGF- $\beta 1$ production by hepatocytes 
and macrophages (17). By contrast, iron depletion inhibits the pancreatic TGF signal, thus inhibiting the phosphorylation of Smad2 (18). Kajikawa et al (19) demonstrated that eicosapentaenoic acid reduces hepatic reactive oxygen species levels and serum ferritin in the methionine- and choline-deficient diet rat model in parallel with hepatic TGF- $\beta 1$. DPP4-I reduced serum ferritin levels, which may lead to reduced levels of hepatic TGF- $\beta 1$ and consequent inhibition of NASH progression.

The present study had several limitations. Firstly, NAFLD and NASH progression were evaluated using ultrasonography and a non-invasive scoring system. The NAFIC score was established to differentiate NASH from NAFL in a cross-sectional study. It is not clear whether the NAFIC score can be used to evaluate longitudinal outcome, which may have resulted in misclassification. Although liver biopsy is the gold standard for the diagnosis of NAFLD and assessment of disease progression, it is unrealistic to perform liver biopsies in all NAFLD patients with type 2 diabetes (20). Secondly, as this was a single arm study with a small number of patients and a short observation period for this type of study, to assessing the effect of significant potential confounding factors, such as calorie intake and exercise status, could not be performed.

In conclusion, DPP4-I administration resulted in decreased NAFIC scores, demonstrating the efficacy of DPP4-I against NAFLD progression. These results indicate that DPP4-I may represent a potential new therapeutic strategy for the prevention of NAFLD progression in NAFLD patients with type 2 diabetes in the future.

\section{Acknowledgements}

Takeda Pharmaceutical Company Limited (Osaka, Japan) supported the present study. The authors would like to thank Mr. Yoshie Nakai and Mr. Hisayo Iino (Third Department of Internal Medicine, Nara Medical University) for their clerical support and Enago (www.enago.jp) for the review of the English language.

\section{References}

1. Angulo P: Nonalcoholic fatty liver disease. N Engl J Med 346: 1221-1231, 2002.

2. Vernon G, Baranova A and Younossi ZM: Systematic review: The epidemiology and natural history of non-alcoholic fatty liver disease and non-alcoholic steatohepatitis in adults. Aliment Pharmacol Ther 34: 274-285, 2011.

3. Friedman SL: Liver fibrosis - from bench to bedside. J Hepatol 38 (Suppl 1): S38-S53, 2003.

4. Fukuhara T, Hyogo H, Ochi H, Fujino H, Kan H, Naeshiro N, Honda Y, Miyaki D, Kawaoka T, Tsuge M, et al: Efficacy and safety of sitagliptin for the treatment of nonalcoholic fatty liver disease with type 2 diabetes mellitus. Hepatogastroenterology 61: 323-328, 2014.

5. Yilmaz Y, Yonal O, Deyneli O, Celikel CA, Kalayci C and Duman DG: Effects of sitagliptin in diabetic patients with nonalcoholic steatohepatitis. Acta Gastroenterol Belg 75: 240-244, 2012
6. Kaji K, Yoshiji H, Ikenaka Y, Noguchi R, Aihara Y, Douhara A, Moriya K, Kawaratani H, Shirai Y, Yoshii J, et al: Dipeptidyl peptidase-4 inhibitor attenuates hepatic fibrosis via suppression of activated hepatic stellate cell in rats. J Gastroenterol 49: 481-491, 2014.

7. Fraser A, Longnecker MP and Lawlor DA: Prevalence of elevated alanine aminotransferase among US adolescents and associated factors: NHANES 1999-2004. Gastroenterology 133: 1814-1820, 2007.

8. Strauss RS, Barlow SE and Dietz WH: Prevalence of abnormal serum aminotransferase values in overweight and obese adolescents. J Pediatr 136: 727-733, 2000.

9. Hamaguchi M, Kojima T, Itoh Y, Harano Y, Fujii K, Nakajima T, Kato T, Takeda N, Okuda J, Ida K, et al: The severity of ultrasonographic findings in nonalcoholic fatty liver disease reflects the metabolic syndrome and visceral fat accumulation. Am J Gastroenterol 102: 2708-2715, 2007.

10. The Committee of the Japan Diabetes Society on the diagnostic criteria of diabetes mellitus. Report of the Committee on the classification and diagnostic criteria of diabetes mellitus. J Diabetes Investig 1: 212-228, 2010.

11. Sumida Y, Yoneda M, Hyogo H, Yamaguchi K, Ono M, Fujii H, Eguchi Y, Suzuki Y, Imai S, Kanemasa K, et al; Japan Study Group of Nonalcoholic Fatty Liver Disease (JSG-NAFLD): A simple clinical scoring system using ferritin, fasting insulin, and type IV collagen $7 \mathrm{~S}$ for predicting steatohepatitis in nonalcoholic fatty liver disease. J Gastroenterol 46: 257-268, 2011.

12. Iwasaki T, Yoneda M, Inamori M, Shirakawa J, Higurashi T, Maeda S, Terauchi Y and Nakajima A: Sitagliptin as a novel treatment agent for non-alcoholic Fatty liver disease patients with type 2 diabetes mellitus. Hepatogastroenterology 58: 2103-2105, 2011.

13. Yilmaz Y, Atug O, Yonal O, Duman D, Ozdogan O, Imeryuz N and Kalayci C: Dipeptidyl peptidase IV inhibitors: Therapeutic potential in nonalcoholic fatty liver disease. Med Sci Monit 15: HY1-HY5, 2009.

14. Mashitani T, Hayashino Y, Okamura S, Kitatani M, Furuya M, Matsunaga S, Kuwata H, Tsujii S and Ishii H: Patient-reported adherence to insulin regimen is associated with glycemic control among Japanese patients with type 2 diabetes: Diabetes Distress and Care Registry at Tenri (DDCRT 3). Diabetes Res Clin Pract 100: 189-194, 2013.

15. Levy MT, McCaughan GW, Abbott CA, Park JE, Cunningham AM, Müller E, Rettig WJ and Gorrell MD: Fibroblast activation protein: A cell surface dipeptidyl peptidase and gelatinase expressed by stellate cells at the tissue remodelling interface in human cirrhosis. Hepatology 29: 1768-1778, 1999.

16. Kaji K, Yoshiji H, Kitade M, Ikenaka Y, Noguchi R, Yoshii J, Yanase K, Namisaki T, Yamazaki M, Moriya K, et al: Impact of insulin resistance on the progression of chronic liver diseases. Int J Mol Med 22: 801-808, 2008.

17. George J, Pera N, Phung N, Leclercq I, Yun Hou J and Farrell G: Lipid peroxidation, stellate cell activation and hepatic fibrogenesis in a rat model of chronic steatohepatitis. J Hepatol 39: 756-764, 2003.

18. Minamiyama Y, Takemura S, Kodai S, Shinkawa H, Tsukioka T, Ichikawa H, Naito Y, Yoshikawa T and Okada S: Iron restriction improves type 2 diabetes mellitus in Otsuka Long-Evans Tokushima fatty rats. Am J Physiol Endocrinol Metab 298: E1140-E1149, 2010.

19. Kajikawa S, Imada K, Takeuchi T, Shimizu Y, Kawashima A, Harada T and Mizuguchi K: Eicosapentaenoic acid attenuates progression of hepatic fibrosis with inhibition of reactive oxygen species production in rats fed methionine- and choline-deficient diet. Dig Dis Sci 56: 1065-1074, 2011.

20. Sumida Y, Nakajima A and Itoh Y: Limitations of liver biopsy and non-invasive diagnostic tests for the diagnosis of nonalcoholic fatty liver disease/nonalcoholic steatohepatitis. World J Gastroenterol 20: 475-485, 2014. 